 \\ TATRA

\section{KURZWEIL-HENSTOCK TYPE INTEGRAL IN FOURIER ANALYSIS ON COMPACT ZERO-DIMENSIONAL GROUP}

\author{
Valentin Skvortsov — Francesco Tulone
}

\begin{abstract}
A Kurzweil-Henstock type integral defined on a zero-dimensional compact abelian group is studied and used to obtain a generalization of some results related to the problem of recovering, by generalized Fourier formulae, the coefficients of convergent series with respect to the characters of such a group.
\end{abstract}

\section{Introduction}

In this paper, we study a Kurzweil-Henstock type integral defined on a zero-dimensional compact abelian group (see [7]) and we use this integral to obtain a generalization of some results related to the problem of recovering, by generalized Fourier formulae, the coefficients of convergent series with respect to the characters of such a group.

In Section 2, we remind some definitions and facts on the structure of a zero-dimensional abelian group and on the theory of the Kurzweil-Henstock integral on such a group. In Section 3, we use the Perron approach to the Kurzweil-Henstock integral to obtain a generalized form of the theorem on recovering the primitive from the derivative. Then, in Section 4, an application to the series with respect to the characters of the above mentioned group is considered. The version of the coefficient problem for the system of characters, we are solving here, is a generalization of the one considered earlier for the Walsh and for the Vilenkin system (see [2]).

In comparison with [7, we are putting some weaker condition on the way of the convergence of the series, but, at the same time, we have to induce some stronger assumption related to the integrability of the sum of the series.

2000 Mathematics Subject Classification: 26A39, 42C10, 43A25, 43A70.

Keywords: compact zero-dimensional abelian group, characters of a group, Kurzweil-Henstock integral, Perron integral, Fourier series, coefficient problem.

Supported by RFFI-08-01-00669 and by NSh-2787.2008.1. 


\section{VALENTIN SKVORTSOV — FRANCESCO TULONE}

\section{Preliminaries}

Consider a zero-dimensional compact abelian group $G$. It is known (see [1]) that a topology in such a group can be given by a chain of subgroups

$$
G=G_{0} \supset G_{1} \supset \ldots \supset G_{n} \supset \ldots,
$$

with $\{0\}=\bigcap_{n=0}^{+\infty} G_{n}$. The subgroups $G_{n}$ are clopen sets with respect to this topology. As $G$ is compact, $G_{n}$ (and so also all its cosets) is compact, and this implies that the quotient groups $G / G_{n}$ and $G_{n} / G_{n+1}$ are finite for each $n$. We denote by $K^{n}, n \geq 0$, any coset of the subgroup $G_{n}$ and, having chosen an element $g \in G$, we denote by $K^{n}(g)$ the coset of the subgroup $G_{n}$ which contains the element $g$, i.e., $K^{n}(g)=g+G_{n}$. For each $g \in G$ the sequence $\left\{K^{n}(g)\right\}$ is decreasing and $\{g\}=\bigcap_{n} K^{n}(g)$.

Let $\mu$ be the Haar measure on the group $G$, normalized so that $\mu(G)=1$. In our case it can be constructed by the usual Lebesgue method starting with a measure on the semi-ring constituted by the family of all cosets $K^{n}$.

We remind definitions of some generalized integrals on the group $G$. First, we introduce the construction of a derivation basis considered in [7]. For any function $\nu: G \rightarrow \mathbb{N}$ we define the set

$$
\beta_{\nu}:=\left\{(I, g): g \in G, I=K^{n}(g), n \geq \nu(g)\right\} .
$$

Then, our derivation basis $\mathcal{B}$ is the family $\left\{\beta_{\nu}\right\}_{\nu}$ where $\nu$ runs over the set of all natural-valued functions on $G$. In the terminology of the derivation basis theory, each coset $K^{n}, n \geq 0$, can be called $\mathcal{B}$-interval of the rank $n$.

This basis has all the usual properties of a general derivation basis (see [4]).

First of all, it has the filter base property:

- $\emptyset \notin \mathcal{B}$,

- for every $\beta_{\nu_{1}}, \beta_{\nu_{2}} \in \mathcal{B}$ there exists $\beta_{\nu} \in \mathcal{B}$ such that $\beta_{\nu} \subset \beta_{\nu_{1}} \cap \beta_{\nu_{2}}$ (it is enough to take $\left.\nu=\max \left\{\nu_{1}, \nu_{2}\right\}\right)$.

Definition 2.1. A $\beta_{\nu}$-partition is a finite collection $\pi$ of elements of $\beta_{\nu}$, where the distinct elements $\left(I^{\prime}, x^{\prime}\right)$ and $\left(I^{\prime \prime}, x^{\prime \prime}\right)$ in $\pi$ have $I^{\prime}$ and $I^{\prime \prime}$ disjoint.

If $L$ is a $\mathcal{B}$-interval and $\bigcup_{(I, x) \in \pi} I=L$ then $\pi$ is called $\beta_{\nu}$-partition of $L$.

Our basis $\mathcal{B}$ has the partitioning property. It means that the following conditions hold:

- for each finite collection $I_{0}, I_{1}, \ldots, I_{n}$ of $\mathcal{B}$-intervals with $I_{1}, \ldots, I_{n} \subset I_{0}$ and $I_{i}, i=1,2, \ldots$, being disjoint, the difference $I_{0} \backslash \bigcup_{i=1}^{n} I_{i}$ can be expressed as a finite union of pairwise disjoint $\mathcal{B}$-intervals;

- for each $\mathcal{B}$-interval $L$ and for any $\beta_{\nu} \in \mathcal{B}$ there exists a $\beta_{\nu}$-partition of $L$. 


\section{KURZWEIL-HENSTOCK TYPE INTEGRAL IN FOURIER ANALYSIS ON GROUPS}

This property of $\mathcal{B}$ follows easily from compactness of any $\mathcal{B}$-interval and from the fact that any two $\mathcal{B}$-intervals $I^{\prime}$ and $I^{\prime \prime}$ are either disjoint or one of them is contained in the other one.

Note that in the case of our basis $\mathcal{B}$, given a point $g \in G$, any $\beta_{\nu \text {-partition }}$ contains only one pair $(I, g)$ with this point $g$.

The upper and the lower $\mathcal{B}$-derivative of a real-valued $\mathcal{B}$-interval function $F$ at a point $g$ are defined as

$$
\bar{D}_{\mathcal{B}} F(g):=\limsup _{n \rightarrow \infty} \frac{F\left(K^{n}(g)\right)}{\mu\left(K^{n}(g)\right)}, \quad \underline{D}_{\mathcal{B}} F(g):=\liminf _{n \rightarrow \infty} \frac{F\left(K^{n}(g)\right)}{\mu\left(K^{n}(g)\right)} .
$$

The $\mathcal{B}$-derivative at $g$ is

$$
D_{\mathcal{B}} F(g):=\lim _{n \rightarrow \infty} \frac{F\left(K^{n}(g)\right)}{\mu\left(K^{n}(g)\right)} .
$$

We say that a $\mathcal{B}$-interval function $F$ is $\mathcal{B}$-continuous at a point $g$, with respect to the basis $\mathcal{B}$, if $\lim _{n \rightarrow \infty} F\left(K^{n}(g)\right)=0$.

The following Kurzweil-Henstock type integral was defined in [7]:

Definition 2.2. A point function (real- or complex-valued) $f$ on a $\mathcal{B}$-interval $L$ is said to be $H_{\mathcal{B}}$-integrable on $L$, with $H_{\mathcal{B}}$-integral $A$, if for every $\varepsilon>0$, there exists a function $\nu: L \mapsto \mathbb{N}$ such that for any $\beta_{\nu}$-partition $\pi$ of $L$ we have:

$$
\left|\sum_{(I, g) \in \pi} f(g) \mu(I)-A\right|<\varepsilon .
$$

We denote the integral value $A$ by $\left(H_{\mathcal{B}}\right) \int_{L} f d \mu$.

We remark that if $f$ is $H_{\mathcal{B}}$-integrable on a $\mathcal{B}$-interval $L$ then it is $H_{\mathcal{B}}$-integrable also on any $\mathcal{B}$-subinterval $K$ of $L$. So, we can define the indefinite integral $F(K)=\left(H_{\mathcal{B}}\right) \int_{K} f d \mu$. It can be checked that the indefinite integral $F$ is a $\mathcal{B}$ -continuous additive $\mathcal{B}$-interval function on the set of all $\mathcal{B}$-subintervals of $L$ and $D_{\mathcal{B}} F(g)=f(g)$ almost everywhere on $L$ (see [7]).

We can also prove that a function equal to zero almost everywhere on $L$ is $H_{\mathcal{B}}$-integrable with zero integral value. Therefore we have

Lemma 2.1. If $f$ is $H_{\mathcal{B}}$-integrable on $L$ and $f=h$ a.e., then $h$ is also $H_{\mathcal{B}^{-}}$ -integrable on $L$ and the integrals coincide.

This justifies the following extension of the definition of $H_{\mathcal{B}}$-integral to the case of functions defined only almost everywhere on $L$. 


\section{VALENTIN SKVORTSOV - FRANCESCO TULONE}

Definition 2.3. A function $f$ defined almost everywhere on a $\mathcal{B}$-interval $L$ is said to be $H_{\mathcal{B}}$-integrable on $L$, with integral value $A$, if the function

$$
f_{1}(g):= \begin{cases}f(g), & \text { where } f \text { is defined } \\ 0, & \text { otherwise }\end{cases}
$$

is $H_{\mathcal{B}}$-integrable on $L$ to $A$ in the sense of Definition 2.2 .

It is clear that a complex-valued function is $H_{\mathcal{B}}$-integrable if and only if both its real and imaginary parts are $H_{\mathcal{B}}$-integrable.

\section{Perron approach to the $H_{\mathcal{B}}$-integral and the problem of recovering a primitive}

Now, we remind the definition of a Perron type integral with respect to the basis $\mathcal{B}$ (see [6]).

Definition 3.1. Let $f$ be a point real-valued function on a $\mathcal{B}$-interval $L$. A $\mathcal{B}$ -interval function $M(m)$ is called a $\mathcal{B}$-major ( $\mathcal{B}$-minor) function of $f$ on $L$ if it is superadditive (subadditive) and the lower (upper) $\mathcal{B}$-derivative satisfies the inequality

$$
\underline{D}_{\mathcal{B}} M(g) \geq f(g) \quad\left(\text { resp. } \bar{D}_{\mathcal{B}} m(g) \leq f(g)\right) \quad \text { for all } \quad g \in L .
$$

A function $f$ is said to be $P_{\mathcal{B}}$-integrable on $L$ if

$$
-\infty<\inf _{M}\{M(L)\}=\sup _{m}\{m(L)\}<+\infty,
$$

where "inf" is taken over all $\mathcal{B}$-major functions of $f$ and "sup" is taken over all $\mathcal{B}$-minor functions of $f$. The common value in (11) is denoted by $\left(P_{\mathcal{B}}\right) \int_{L} f$ and is called $P_{\mathcal{B}}$-integral of $f$ on $L$.

If $f$ is $P_{\mathcal{B}}$-integrable on a $\mathcal{B}$-interval $L$ then it is $P_{\mathcal{B}}$-integrable also on any $\mathcal{B}$-subinterval $K$ of $L$, and (11) holds for this $K$.

As in the case of a general derivation basis (see [4]), it can be checked that, for our basis $\mathcal{B}$, the $H_{\mathcal{B}}$-integral is equivalent to the $P_{\mathcal{B}}$-integral.

The following theorem was actually proved in [6].

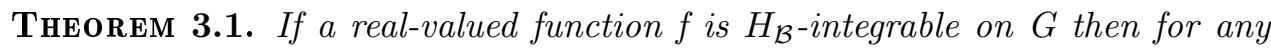
$\varepsilon>0$ there exist a $\mathcal{B}$-continuous $\mathcal{B}$-major function $M$ and a $\mathcal{B}$-continuous $\mathcal{B}$ -minor function $m$ of $f$ such that $M(G)-m(G)<\varepsilon$.

We note that, as a by-product of this theorem, we get that the Perron type integral, with respect to our basis, defined by means of $\mathcal{B}$-continuity major and minor functions is equivalent to the one defined without the assumption of $\mathcal{B}$ continuity of major and minor functions. 


\section{KURZWEIL-HENSTOCK TYPE INTEGRAL IN FOURIER ANALYSIS ON GROUPS}

In order to prove the next theorem, we need several auxiliary statements. The first one is the following

Lемма 3.1. If for a superadditive $\mathcal{B}$-interval function $\phi$ and for some $\mathcal{B}$-interval $K^{n}$ we have $\phi\left(K^{n}\right) \leq a \mu\left(K^{n}\right)$ for some real number $a$, then we can find at least one $\mathcal{B}$-interval $K^{n+1} \subset K^{n}$ such that $\phi\left(K^{n+1}\right) \leq a \mu\left(K^{n+1}\right)$.

Pr o of. Let $K^{n}=\cup_{i=1}^{t} K_{i}^{n+1}$. Supposing that $\phi\left(K_{i}^{n+1}\right)>a \mu\left(K_{i}^{n+1}\right)$ for all $i=1, \ldots, t$ and using the superadditivity of $\phi$, we have

$$
\phi\left(K^{n}\right) \geq \sum_{i=1}^{t} \phi\left(K_{i}^{n+1}\right)>a \sum_{i=1}^{t} \mu\left(K_{i}^{n+1}\right)=a \mu\left(K^{n}\right)
$$

getting a contradiction.

Lemma 3.2. Let $\phi$ be a superadditive $\mathcal{B}$-interval function. If $\bar{D}_{\mathcal{B}} \phi(g) \geq 0$ everywhere on $G$ except on a countable set $S$, where $\phi$ satisfies the inequality

$$
\liminf _{n \rightarrow \infty} \phi\left(K^{n}(g)\right) \leq 0 \leq \limsup _{n \rightarrow \infty} \phi\left(K^{n}(g)\right)
$$

then $\phi(K) \geq 0$ for any $\mathcal{B}$-interval $K$.

Pr o of. Suppose that there exists a $\mathcal{B}$-interval $K^{n}$ such that $\frac{\phi\left(K^{n}\right)}{\mu\left(K^{n}\right)}=a<0$. Having assumed this, we show that, for a certain $m>n$, there exist at least two $\mathcal{B}$-intervals $K_{1}^{m}$ and $K_{2}^{m}$ such that

$$
\frac{\phi\left(K^{r}\right)}{\mu\left(K^{r}\right)} \leq a
$$

for every $K^{r}$, where $K_{1}^{m} \subset K^{r} \subset K^{n}$ or $K_{2}^{m} \subset K^{r} \subset K^{n}$ with $n<r \leq m$. Suppose this is not true. Let $K^{n}=\cup_{i=1}^{t} K_{i}^{n+1}$ and let $K_{1}^{n+1}$ be the only $\mathcal{B}$-interval of rank $n+1$ for which, according to Lemma 3.1 the inequality $\phi\left(K_{1}^{n+1}\right) \leq a \mu\left(K_{1}^{n+1}\right)$ holds while for the rest of $\mathcal{B}$-intervals $K_{j}^{n+1}, j=2, \ldots, t$, the opposite inequality $\phi\left(K_{j}^{n+1}\right)>a \mu\left(K_{j}^{n+1}\right)$ holds. Then, by superadditivity of $\phi$, we obtain

$$
\begin{aligned}
\phi\left(K_{1}^{n+1}\right) & \leq \phi\left(K^{n}\right)-\sum_{j=2}^{t} \phi\left(K_{j}^{n+1}\right) \\
& <a \mu\left(K^{n}\right)-a \sum_{j=2}^{t} \mu\left(K_{j}^{n+1}\right) \\
& =a\left(\mu\left(K^{n}\right)-\sum_{j=2}^{t} \mu\left(K_{j}^{n+1}\right)\right) \\
& =a \mu\left(K_{1}^{n+1}\right),
\end{aligned}
$$




\section{VALENTIN SKVORTSOV — FRANCESCO TULONE}

getting that $b=\phi\left(K_{1}^{n+1}\right)-a \mu\left(K_{1}^{n+1}\right)<0$. In the next step, in the same way, we can choose $K_{1}^{n+2} \subset K_{1}^{n+1}$ for which we have

$$
\begin{aligned}
& \phi\left(K_{1}^{n+2}\right)-a \mu\left(K_{1}^{n+2}\right) \\
& \leq \phi\left(K_{1}^{n+1}\right)-\sum_{j=2}^{p} \phi\left(K_{j}^{n+2}\right)-a \mu\left(K_{1}^{n+1}\right)+a \sum_{j=2}^{p} \mu\left(K_{j}^{n+2}\right) \\
& \leq b .
\end{aligned}
$$

Now, proceeding by induction, we construct a decreasing sequence of $\mathcal{B}$-intervals $\left\{K_{1}^{n+s}\right\}_{s=1}^{+\infty}$ converging to a point $g$ such that (3) holds for any $r=n+s \geq n$, and at the same time,

$$
\phi\left(K_{1}^{n+s}\right)-a \mu\left(K_{1}^{n+s}\right) \leq b<0 .
$$

Therefore, $\bar{D}_{\mathcal{B}} \phi(g) \leq a<0$, so $g \in S$. On the other, hand passing to the limit in the inequality (4) when $s \rightarrow \infty$, we obtain $\limsup _{s \rightarrow \infty} \phi\left(K_{1}^{n+s}\right) \leq b<0$ and this obviously contradicts the inequality (2) at the points of the set $S$. So, we have proved the existence of at least two $\mathcal{B}$-intervals satisfying the property described in (3).

For each of this two $\mathcal{B}$-intervals obtained above, we repeat the previous argument getting four $\mathcal{B}$-intervals with the corresponding property. Proceeding by induction, we obtain a continuum set of decreasing sequences of $\mathcal{B}$-intervals convergent to a continuum set of points $g$ where $\bar{D}_{\mathcal{B}} \phi(g)<a$. This is in contradiction with the countability of the set $S$.

Using Theorem 3.1, we obtain the following theorem on recovering a primitive.

TheOREM 3.2. Let an additive real-valued $\mathcal{B}$-interval function $F$ and $a H_{\mathcal{B}^{-}}$ -integrable real-valued function $f$ satisfy the inequality

$$
\underline{D}_{\mathcal{B}} F(g) \leq f(g) \leq \bar{D}_{\mathcal{B}} F(g)
$$

everywhere on $G$ except on a countable set $S$, where

$$
\liminf _{n \rightarrow \infty} F\left(K^{n}(g)\right) \leq 0 \leq \limsup _{n \rightarrow \infty} F\left(K^{n}(g)\right) .
$$

Then $D_{\mathcal{B}}(F(g))=f(g)$ a.e. on $G$ and $F$ is the indefinite $H_{\mathcal{B}}$-integral of $f$.

Pr o of. Since $f$ is $H_{\mathcal{B}}$-integrable on $G$ then, by Theorem 3.1 for a fixed $\varepsilon>0$ there exist a $\mathcal{B}$-continuous $\mathcal{B}$-major function $M_{\varepsilon}$ and a $\mathcal{B}$-continuous $\mathcal{B}$-minor function $m_{\varepsilon}$ such that

$$
\bar{D}_{\mathcal{B}} m_{\varepsilon}(g) \leq f(g) \leq \underline{D}_{\mathcal{B}} M_{\varepsilon}(g)
$$

and

$$
M_{\varepsilon}(G)-m_{\varepsilon}(G)<\varepsilon .
$$


Let $\Phi(K)=\left(H_{\mathcal{B}}\right) \int_{K} f d \mu$ be the indefinite $H_{\mathcal{B}}$-integral of $f$. In view of the equivalence of the $H_{\mathcal{B}}$-integral and $P_{\mathcal{B}}$-integral, we have (see (1))

$$
\Phi(K)=\inf _{M}\{M(K)\}=\sup _{m}\{m(K)\} .
$$

So, we get

$$
M_{\varepsilon}(K) \leq \Phi(K)+\varepsilon \quad \text { and } \quad \Phi(K)-\varepsilon \leq m_{\varepsilon}(K)
$$

for every $\mathcal{B}$-interval $K$.

From (7) and from the assumption (5), we obtain

$$
\bar{D}_{\mathcal{B}}\left(M_{\varepsilon}(g)-F(g)\right) \geq \underline{D}_{\mathcal{B}} M_{\varepsilon}(g)-\underline{D}_{\mathcal{B}} F(g) \geq \underline{D}_{\mathcal{B}} M_{\varepsilon}(g)-f(g) \geq 0
$$

if $g \in G \backslash S$.

Note that $\mathcal{B}$-continuity of $M_{\varepsilon}$ means that $\lim _{n \rightarrow+\infty} M_{\varepsilon}\left(K^{n}(g)\right)=0$ for every $g \in G$. Combining this with ([6), we obtain for each $g \in S$

$$
\begin{aligned}
& \liminf _{n \rightarrow \infty}\left(M_{\varepsilon}\left(K^{n}(g)\right)-F\left(K^{n}(g)\right)\right) \\
& =\lim _{n \rightarrow \infty} M_{\varepsilon}\left(K^{n}(g)\right)-\limsup _{n \rightarrow \infty} F\left(K^{n}(g)\right) \\
& \leq 0 \\
& \leq \lim _{n \rightarrow \infty} M_{\varepsilon}\left(K^{n}(g)\right)-\liminf _{n \rightarrow \infty} F\left(K^{n}(g)\right) \\
& =\limsup _{n \rightarrow \infty}\left(M_{\varepsilon}\left(K^{n}(g)\right)-F\left(K^{n}(g)\right)\right) .
\end{aligned}
$$

So, all the assumptions of Lemma 3.2 are fulfilled for the difference $M_{\varepsilon}-F$ and we get $M_{\varepsilon}(K)-F(K) \geq 0$ for any $\mathcal{B}$-interval $K$. In a similar way, we prove that $m_{\varepsilon}(K)-F(K) \leq 0$ for any $\mathcal{B}$-interval $K$. Therefore, we obtain

$$
\Phi(K)-\varepsilon \leq m_{\varepsilon}(K) \leq F(K) \leq M_{\varepsilon}(K) \leq \Phi(K)+\varepsilon .
$$

Since $\varepsilon$ is arbitrary, we have $\Phi(K)=F(K)$ for any $\mathcal{B}$-interval $K$. Moreover, the $\mathcal{B}$-differentiability of the $H_{\mathcal{B}}$-integral implies $D_{\mathcal{B}}(F(g))=f(g)$ a.e. on $G$ completing the proof.

\section{Application to the series with respect to the characters}

Let $\Gamma$ denote the dual group of $G$, i.e., the group of characters of the group $G$. It is known (see [1]) that under the assumptions imposed on $G$, the group $\Gamma$ is a discrete abelian group with respect to the pointwise multiplication of characters.

Moreover, we can represent it as a sum of an increasing sequence of subgroups of finite order:

$$
\Gamma_{0} \subset \Gamma_{-1} \subset \Gamma_{-2} \subset \ldots \subset \Gamma_{-n} \subset \ldots
$$




\section{VALENTIN SKVORTSOV — FRANCESCO TULONE}

Then, $\Gamma=\cup_{i=0}^{+\infty} \Gamma_{-i}$ and $\cap_{i=0}^{+\infty} \Gamma_{-i}=\left\{\gamma^{(0)}\right\}$, where $\gamma^{(0)}(g)=1$ for all $g \in G$. For each $n \geq 0$ the group $\Gamma_{-n}$ is the annulator of $G_{n}$, i.e.,

$$
\Gamma_{-n}=G_{n}^{\perp}:=\left\{\gamma \in \Gamma: \gamma(g)=1 \text { for all } g \in G_{n}\right\} .
$$

The following lemma can be easily proved (see [7]).

LEMmA 4.1. If $\gamma \in \Gamma_{-n}$ then $\gamma$ is constant on each coset $K^{n}$ of $G_{n}$.

The characters $\gamma$ constitute a countable orthonormal system on $G$ with respect to the Haar measure $\mu$ (see [1]), and we can consider series

with respect to this system.

$$
\sum_{\gamma \in \Gamma} a_{\gamma} \gamma
$$

We define a convergence of this series at a point $g$ as the convergence of its partial sums of the form

$$
S_{n}(g):=\sum_{\gamma \in \Gamma_{-n}} a_{\gamma} \gamma(g)
$$

when $n$ tends to infinity.

We associate with the series $\sum_{\gamma \in \Gamma} a_{\gamma} \gamma$ a complex-valued set function $F$ defined on each coset $K^{n}$ by

$$
F\left(K^{n}\right):=\int_{K^{n}} S_{n}(g) d \mu .
$$

The above integral can be understood in the Lebesgue sense. It can be checked that $F$ is an additive function on the family of all $\mathcal{B}$-intervals.

Since by Lemma 4.1 the sum $S_{n}$ is constant on each $K^{n}$, then the definition of $F\left(K^{n}\right)$ implies

$$
S_{n}(g)=\frac{F\left(K^{n}(g)\right)}{\mu\left(K^{n}(g)\right)} .
$$

As a direct consequence of the above equality, we get the following ones

$$
\begin{array}{ll}
\liminf _{n \rightarrow \infty} \operatorname{Re} S_{n}(g)=\underline{D}_{\mathcal{B}} \operatorname{Re} F(g), & \limsup _{n \rightarrow \infty} \operatorname{Re} S_{n}(g)=\bar{D}_{\mathcal{B}} \operatorname{Re} F(g), \\
\liminf _{n \rightarrow \infty} \operatorname{Im} S_{n}(g)=\underline{D}_{\mathcal{B}} \operatorname{Im} F(g), & \limsup _{n \rightarrow \infty} \operatorname{Im} S_{n}(g)=\bar{D}_{\mathcal{B}} \operatorname{Im} F(g) .
\end{array}
$$

The next theorem is a generalization of a similar one for the case of the Lebesgue integral and the Vilenkin-Price system (see [3, Theorem 2.8.1]).

TheOREM 4.1. A series $\sum_{\gamma \in \Gamma} a_{\gamma} \gamma$ is the $H_{\mathcal{B}}$-Fourier series of some $H_{\mathcal{B}}$-integrable function $f$ if the function $F$ associated by (8) with this series coincides on each $\mathcal{B}$-interval $K$ with the indefinite integral $\left(H_{\mathcal{B}}\right) \int_{K} f d \mu$. 
Pr o of. We have $F(K)=\left(H_{\mathcal{B}}\right) \int_{K} f$ for any $\mathcal{B}$-interval $K$. Fix a character $\gamma$ and choose $n$ such that $\gamma \in \Gamma_{-n}$. For this $n$ the group $G$ can be represented as a finite union $G=\cup_{i} K_{i}^{n}$. By Lemma 4.1, $\gamma$ and also the sum $S_{n}$ are constant on each $K_{i}^{n}$. Let

$$
S_{n}(g)=s_{i} \quad \text { and } \quad \gamma(g)=\xi_{i} \quad \text { if } \quad g \in K_{i}^{n}
$$

Then we get

$$
\begin{aligned}
a_{\gamma}=\int_{G} S_{n} \gamma d \mu & =\sum_{i} s_{i} \xi_{i} \mu\left(K_{i}^{n}\right)=\sum_{i} \xi_{i} F\left(K_{i}^{n}\right) \\
& =\sum_{i} \xi_{i}\left(H_{\mathcal{B}}\right) \int_{K_{i}^{n}} f d \mu=\sum_{i}\left(H_{\mathcal{B}}\right) \int_{K_{i}^{n}} f \gamma d \mu=\left(H_{\mathcal{B}}\right) \int_{G} f \gamma d \mu .
\end{aligned}
$$

Now, we apply Theorem 3.2 and Theorem 4.1 to get the following result about recovering the coefficients by generalized Fourier formulae:

TheOREM 4.2. Suppose that the partial sums $S_{n}(g)$ of a series $\sum_{\gamma \in \Gamma} a_{\gamma} \gamma$ and an $H_{\mathcal{B}}$-integrable function $f$ satisfy the inequalities

$$
\begin{aligned}
& \liminf _{n \rightarrow \infty} \operatorname{Re} S_{n}(g) \leq \operatorname{Re} f(g) \leq \limsup _{n \rightarrow \infty} \operatorname{Re} S_{n}(g), \\
& \liminf _{n \rightarrow \infty} \operatorname{Im} S_{n}(g) \leq \operatorname{Im} f(g) \leq \limsup _{n \rightarrow \infty} \operatorname{Im} S_{n}(g)
\end{aligned}
$$

everywhere on $G$ except on a countable set $S$, where

$$
\begin{aligned}
& \liminf _{n \rightarrow \infty} \mu\left(K^{n}\right) \operatorname{Re} S_{n}(g) \leq 0 \leq \limsup _{n \rightarrow \infty} \mu\left(K^{n}\right) \operatorname{Re} S_{n}(g), \\
& \liminf _{n \rightarrow \infty} \mu\left(K^{n}\right) \operatorname{Im} S_{n}(g) \leq 0 \leq \limsup _{n \rightarrow \infty} \mu\left(K^{n}\right) \operatorname{Im} S_{n}(g)
\end{aligned}
$$

hold. Then the series $\sum_{\gamma \in \Gamma} a_{\gamma} \gamma$ is convergent to $f$ a.e. and it is the $H_{\mathcal{B}}$-Fourier series of $f$.

P r o of. Let $F$ be defined by (8). The conditions (9), (11) and (13) imply that the assumptions of Theorem 3.2 are fulfilled for $\operatorname{Re} F$. Then $D_{\mathcal{B}} \operatorname{Re} F(g)=$ $\operatorname{Re} f(g)$ a.e. and $\operatorname{Re} F(K)=\left(H_{\mathcal{B}}\right) \int_{K} \operatorname{Re} f d \mu$ for all $\mathcal{B}$-intervals $K$. In a similar way, by (10), (12) and (14), we get $D_{\mathcal{B}} \operatorname{Im} F(g)=\operatorname{Im} f(g)$ a.e. and $\operatorname{Im} F(K)=$ $\left(H_{\mathcal{B}}\right) \int_{K} \operatorname{Im} f d \mu$. So, $D_{\mathcal{B}} F(g)=f(g)$ a.e. and $F(K)=\left(H_{\mathcal{B}}\right) \int_{K} f d \mu$. Once again, using (9) and (10), we get that $S_{n}$ is convergent a.e. to $f$ and, by Theorem 4.1, this series is the $H_{\mathcal{B}}$-Fourier series of $f$. 


\section{VALENTIN SKVORTSOV - FRANCESCO TULONE}

Corollary 4.3. Suppose that the partial sums $S_{n}(g)$ of a series $\sum_{\gamma \in \Gamma} a_{\gamma} \gamma$ and an $H_{\mathcal{B}}$-integrable function $f$ satisfy the inequalities

$$
\begin{aligned}
& \liminf _{n \rightarrow \infty} \operatorname{Re} S_{n}(g) \leq \operatorname{Re} f(g) \leq \limsup _{n \rightarrow \infty} \operatorname{Re} S_{n}(g), \\
& \liminf _{n \rightarrow \infty} \operatorname{Im} S_{n}(g) \leq \operatorname{Im} f(g) \leq \limsup _{n \rightarrow \infty} \operatorname{Im} S_{n}(g)
\end{aligned}
$$

a.e. on $G$ and the condition

$$
\limsup \left|S_{n}(g)\right|<\infty
$$

everywhere on $G$ except on a countable set $S$, where (13) and (14) hold. Then the series $\sum_{\gamma \in \Gamma} a_{\gamma} \gamma$ is convergent to $f$ a.e. and it is the $H_{\mathcal{B}}$-Fourier series of $f$.

Proof. Denote by $E$ the set where the inequality (15) or (16) does not hold. Then it is enough to notice that the function $\phi$ defined as

$$
\phi(g):= \begin{cases}f(g) & \text { if } g \in G \backslash E, \\ \limsup _{n \rightarrow \infty} \operatorname{Re} S_{n}(g)+i \limsup _{n \rightarrow \infty} \operatorname{Im} S_{n}(g) & \text { if } g \in E \backslash S\end{cases}
$$

satisfies the assumption of Theorem 4.2.

We remark that Theorem 4.2 is not true if we replace $H_{\mathcal{B}}$-integrability of the function $f$ with Denjoy-Khintchine integrability (see [5]), but it also becomes true in the Denjoy-Khintchine case if we put some additional hypothesis on the group and on the type of convergence (see [8] ).

In the conclusion we note that if we put some stronger assumption on the convergence of the series in the last theorem, we get a similar result in which the a priori assumption of $H_{\mathcal{B}}$-integrability of $f$ can be dropped (see [7]).

\section{REFERENCES}

[1] AGAEV, G. N.-VILENKIN, N. YA.-DZHARARLI, G. M.-RUBinshteJn, A. I.: Multiplicative System of Functions and Harmonic Analysis on Zero-Dimensional Groups. "Ehlm", Baku, 1981. (In Russian)

[2] BOKAEV, N. A-SKVORTSOV, V.: Generalization of uniqueness theorem for series in multiplicative system, Vestnik Moskov. Univ. Ser. I Mat. Mekh. 1987 (1987), 11-15; Engl. transl. in Moscow Univ. Math. Bull. 42 (1987), 11-16.

[3] GOLUBOV, B.-EFIMOV, A.-SKVORTSOV, V.: Walsh Series and Transforms. Theory and Applications. Kluwer Acad. Publ., Dordrecht, 1991.

[4] OSTASZEWSKI, K. M.: Henstock Integration in the Plane, in: Mem. Amer. Math. Soc., Vol. 63, no. 353, AMS, Providence, RI, 1986. 


\section{KURZWEIL-HENSTOCK TYPE INTEGRAL IN FOURIER ANALYSIS ON GROUPS}

[5] SKVORTSOV, V.-KOROLEVA, M. P.: Series in multiplicative systems convergent to Denjoy-integrable functions, Mat. Sb. 186 (1995), 129-150; Engl. transl. Sb. Math. 186 (1995), 1821-1842.

[6] SKVORTSOV, V.-TUlONE, F.: Perron type integral on compact zero-dimensional Abelian groups, Vestnik Moskov. Univ. Ser. I Mat. Mekh. 63 (2008), 37-42; Engl. transl. Moscow Univ. Math. Bull. 63 (2008), 119-124.

[7] SKVORTSOV, V.-TULONE, F.: Kurzweil-Henstock type integral on zero-dimensional group and some of its applications, Czechoslovak Math. J. 58 (2008), 1167-1183.

[8] TULONE, F.: Denjoy and $\mathcal{P}$-path integrals on compact groups in an inversion formula for multiplicative transforms, Tatra Mt. Math. Publ. 42 (2008), 27-37.

Received November 20, 2008

\author{
Valentin Skvortsov \\ Institute of Mathematics \\ Casimirus the Great University \\ pl. Weyssenhoffa 11 \\ PL-85-072 Bydgoszcz \\ POLAND
}

Department of Mathematics

Moscow State University

$R U-119991$ Moscow

RUSSIA

E-mail: vaskvor2000@yahoo.com

Francesco Tulone

Dipartimento di Matematica ed Applicazioni Università di Palermo

via Archirafi 34

IT-90123 Palermo

ITALY

E-mail: tulone@math.unipa.it 